PROCEEDINGS OF THE

AMERICAN MATHEMATICAL SOCIETY

Volume 138, Number 1, January 2010, Pages 375-384

S 0002-9939(09)10069-2

Article electronically published on September 3, 2009

\title{
CONSTRUCTING KNOT TUNNELS USING GIANT STEPS
}

\author{
SANGBUM CHO AND DARRYL MCCULLOUGH
}

(Communicated by Daniel Ruberman)

\begin{abstract}
In 2000, Goda, Scharlemann, and Thompson described a general construction of all tunnels of tunnel number 1 knots using "tunnel moves". The theory of tunnels introduced by Cho and McCullough provides a combinatorial approach to understanding tunnel moves. We use it to calculate the number of distinct minimal sequences of such moves that can produce a given tunnel. As a consequence, we see that for a sparse infinite set of tunnels, the minimal sequence is unique, but generically a tunnel will have many such constructions. Finally, we give a characterization of the tunnels with a unique minimal sequence.
\end{abstract}

\section{INTRODUCTION}

In previous work [3], we introduced a theory of tunnels of tunnel number 1 knots based on the disk complex of the genus- 2 handlebody. It provides a simplicial complex $\mathcal{D}(H) / \mathcal{G}$ whose vertices correspond to the (equivalence classes of) tunnels of all tunnel number 1 knots. As we will explain below, two tunnels span a 1simplex of $\mathcal{D}(H) / \mathcal{G}$ exactly when each is obtained from the other by a construction given by H. Goda, M. Scharlemann, and A. Thompson in 6. For reasons that will become apparent, we call these constructions "giant steps". The connectivity of $\mathcal{D}(H) / \mathcal{G}$ shows that every tunnel can be obtained from the unique tunnel $\pi_{0}$ of the trivial knot by some sequence of giant steps, a fact already proved in 6 .

In this work, we will use the combinatorial structure of $\mathcal{D}(H) / \mathcal{G}$ to examine minimal length sequences of giant steps that start from $\pi_{0}$ and produce a given tunnel $\tau$. Our main result is an algorithm to calculate the number of distinct such sequences. In fact, this is just the number of shortest paths between two vertices of the Farey graph. Although the algorithm is quite elementary, we have been unable to find it in the literature. It is effective and we have implemented it computationally [5. We will use it here to see that for a sparse infinite set of tunnels, the minimal construction sequence is unique, but generically a tunnel will have many such sequences. In Proposition 4.4, we give a characterization of the tunnels with unique minimal construction sequence.

The length of a minimal sequence of giant steps producing a given tunnel is equal to the invariant called the depth of the tunnel, defined below. This invariant is used extensively in our work on bridge numbers of tunnel number 1 knots in [4].

Received by the editors July 29, 2008, and, in revised form, May 8, 2009.

2000 Mathematics Subject Classification. Primary 57M25.

The research of both authors was supported in part by NSF grant DMS-0802424.

(C)2009 American Mathematical Society Reverts to public domain 28 years from publication 


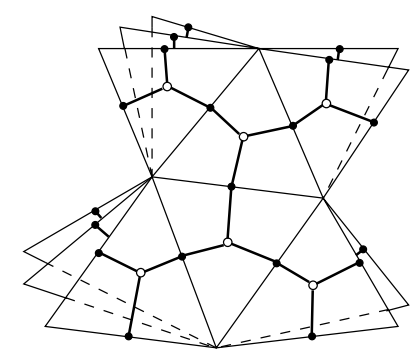

Figure 1. A portion of the nonseparating disk complex $\mathcal{D}(H)$ and the tree $\widetilde{\mathcal{T}}$. Countably many 2 -simplices meet along each edge.

Only a minimal amount of the theory from $[3]$ is needed for the present application. We review it briefly in Sections 1 and 2. Section 3 defines giant steps precisely, and Section 4 presents the algorithm and some of its uses.

We thank the referee for a careful reading of the manuscript and for suggesting that we prove a characterization such as Proposition 4.4

\section{The TREE OF KNOT TUNNELS}

Let $H$ be a genus-2 orientable handlebody, regarded as the standard unknotted handlebody in $S^{3}$. For us, a disk in $H$ means a properly imbedded disk in $H$, which is assumed to be nonseparating unless otherwise stated. The disk complex $\mathcal{D}(H)$ is a 2-dimensional, contractible simplicial complex, whose vertices are the isotopy classes of disks in $H$, such that a collection of $k+1$ vertices spans a $k$ simplex if and only if they admit a set of pairwise-disjoint representatives. Each 1-simplex of $\mathcal{D}(H)$ is contained in countably many 2-simplices. As suggested by Figure 1. $\mathcal{D}(H)$ grows outward from any of its 2-simplices in a treelike way. In fact, it deformation retracts to the tree $\widetilde{\mathcal{T}}$ seen in Figure 1, which is the subcomplex of the first barycentric subdivision $\mathcal{D}^{\prime}(H)$ spanned by the barycenters of 1-simplices and 2-simplices of $\mathcal{D}(H)$. We refer the reader to [3] for details on these objects.

A tunnel of a tunnel number 1 knot produces a disk in $H$ as follows. The tunnel is a 1-handle attached to a regular neighborhood of the knot to form an unknotted genus-2 handlebody. An isotopy moving this handlebody to $H$ carries a cocore 2 disk of that 1-handle to a nonseparating disk in $H$ and carries the tunnel number 1 knot to a core circle of the solid torus obtained by cutting $H$ along that disk.

The indeterminacy in the choice of the isotopy is the group of isotopy classes of orientation-preserving homeomorphisms of $S^{3}$ that preserve $H$. This group is called the Goeritz group $\mathcal{G}$. Work of M. Scharlemann [7] and E. Akbas [1 proves that $\mathcal{G}$ is finitely presented and even provides a simple presentation of it.

Since two disks in $H$ determine equivalent tunnels exactly when they differ by an isotopy moving $H$ through $S^{3}$, the collection of all (equivalence classes of) tunnels of all tunnel number 1 knots corresponds to the set of orbits of vertices of $\mathcal{D}(H)$ under $\mathcal{G}$. So it is natural to examine the quotient complex $\mathcal{D}(H) / \mathcal{G}$, which is illustrated in Figure 2 .

Through work of the first author [2], the action of $\mathcal{G}$ on $\mathcal{D}(H)$ is well-understood. A primitive disk in $H$ is a disk $D$ such that there is a disk $E$ in $\overline{S^{3}-H}$ for which $\partial D$ and $\partial E$ intersect transversely in one point in $\partial H$. The primitive disks (regarded 


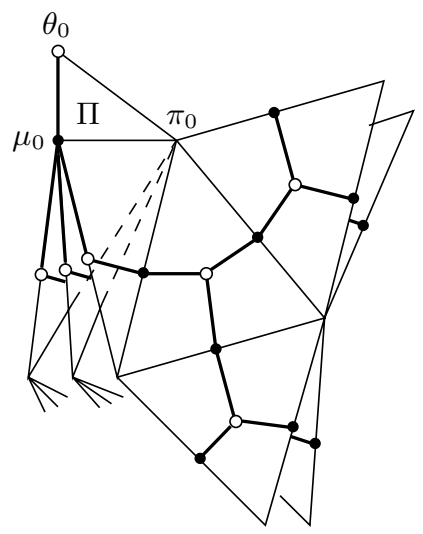

Figure 2. A portion of $\mathcal{D}(H) / \mathcal{G}$ and $\mathcal{T}$ near the primitive orbits.

as vertices) span a contractible subcomplex $\mathcal{P}(H)$ of $\mathcal{D}(H)$, called the primitive subcomplex. The action of $\mathcal{G}$ on $\mathcal{P}(H)$ is as transitive as possible; indeed, the quotient $\mathcal{P}(H) / \mathcal{G}$ is a single 2 -simplex $\Pi$ which is the image of any 2 -simplex of the first barycentric subdivision of $\mathcal{P}(H)$. Its vertices are $\pi_{0}$, the orbit of all primitive disks, $\mu_{0}$, the orbit of all pairs of disjoint primitive disks, and $\theta_{0}$, the orbit of all triples of disjoint primitive disks. Regarded as a tunnel, $\pi_{0}$ is the unique tunnel of the trivial knot.

On the remainder of $\mathcal{D}(H)$, the stabilizers of the action are as small as possible. A 2-simplex which has two primitive vertices and one nonprimitive is identified with some other such simplices, then folded in half and attached to $\Pi$ along the edge $\left\langle\mu_{0}, \pi_{0}\right\rangle$. The nonprimitive vertices of such 2 -simplices are exactly the disks in $\mathcal{D}(H)$ that are disjoint from some primitive pair, and these are called simple disks. As tunnels, they are the upper and lower tunnels of 2-bridge knots, and we call them the simple tunnels. The remaining 2 -simplices of $\mathcal{D}(H)$ receive no self-identifications, and they descend to portions of $\mathcal{D}(H) / \mathcal{G}$ that are treelike and are attached to one of the edges $\left\langle\pi_{0}, \tau_{0}\right\rangle$ where $\tau_{0}$ is simple.

The tree $\widetilde{\mathcal{T}}$ shown in Figure 1 is constructed as follows. Let $\mathcal{D}^{\prime}(H)$ be the first barycentric subdivision of $\mathcal{D}(H)$. Denote by $\widetilde{\mathcal{T}}$ the subcomplex of $\mathcal{D}^{\prime}(H)$ obtained by removing the open stars of the vertices of $\mathcal{D}(H)$. It is a bipartite graph, with "white" vertices of valence 3 represented by triples and "black" vertices of (countably) infinite valence represented by pairs. The valences reflect the fact that moving along an edge from a triple to a pair corresponds to removing one of its three disks, while moving from a pair to a triple corresponds to adding one of infinitely many possible third disks to a pair.

The image $\widetilde{\mathcal{T}} / \mathcal{G}$ of $\widetilde{\mathcal{T}}$ in $\mathcal{D}^{\prime}(H) / \mathcal{G}$ is a tree $\mathcal{T}$. The vertices of $\mathcal{D}^{\prime}(H) / \mathcal{G}$ that are images of vertices of $\mathcal{D}(H)$ are not in $\mathcal{T}$, but their links in $\mathcal{D}^{\prime}(H) / \mathcal{G}$ are subcomplexes of $\mathcal{T}$. These links are infinite trees. For each such vertex $\tau$ of $\mathcal{D}^{\prime}(H) / \mathcal{G}$, i.e. each tunnel, there is a unique shortest path in $\mathcal{T}$ from $\theta_{0}$ to the vertex in the link of $\tau$ that is closest to $\theta_{0}$. This path is called the principal path of $\tau$, and this closest vertex is a triple, called the principal vertex of $\tau$. The two disks in the principal vertex, other than $\tau$, are called the principal pair of $\tau$. They are exactly the disks called $\mu^{+}$and $\mu^{-}$that play a key role in 8 . Figure 3 shows the principal path of a certain tunnel. 


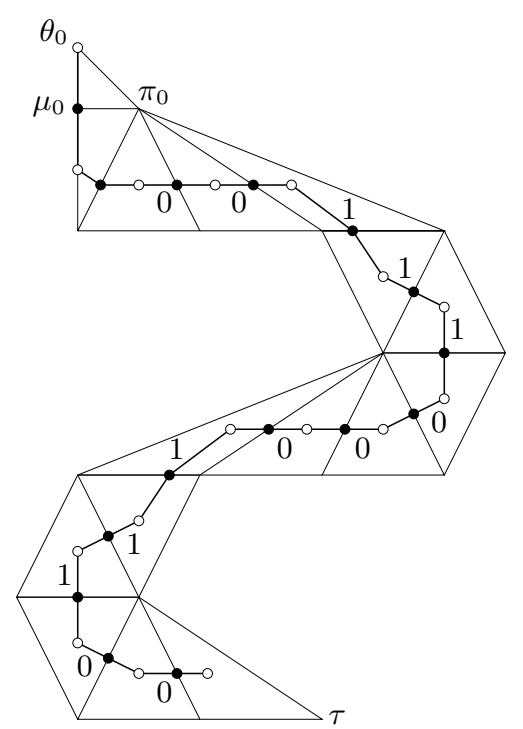

FiguRE 3. The principal path of a tunnel $\tau$ having binary invariants 0011100011100 or, equivalently, with step sequence "DRRRDRDLLLDLDRR".

\section{THE BINARY INVARIANTS}

Moving through the tree $\mathcal{T} / \mathcal{G}$ corresponds to a useful construction of tunnels, called the cabling construction, but we will not need it here. What is important for us is the combinatorial structure near the principal path of $\tau$. This structure is determined by a sequence of "binary" invariants $s_{2}, s_{3}, \ldots, s_{n}$, defined in $[3$. We do not need their formal definition, which involves the cabling construction, for we can think of them in a very simple way, from the viewpoint of a traveler along the path. A step of the principal path is a portion between successive white vertices. At Step 0, a traveler goes from $\theta_{0}$ to the principal vertex of some simple tunnel. At Step 1, the traveler (whom we are viewing from above) must make a left turn. Starting with Step 2, the traveler must make a choice of turning left or turning right out of the white vertex. The invariant $s_{2}$ is 0 if this is a left turn and 1 if it is a right turn. In general, $s_{i}$ is 0 if the direction of the turn at Step $i$ is the same as the direction of the turn at Step $i-1$, and 1 if it is different. Figure 3 shows the principal path of a tunnel with binary invariants 0011100011100.

It is sometimes useful to describe the principal path from the reader's viewpoint. The initial step is always down ("D") and the second step, due to the standard way that we draw the picture, is to the reader's right ("R"). Each subsequent step is either left ("L"), down, or right. An "L" can only be followed by another "L" or a "D", according as the corresponding binary invariant $s$ is 0 or 1 , and similarly an "R" is followed by another "R" or a "D", according as $s$ is 0 or 1 . When the previous step is " $\mathrm{D}$ ", then the effect of $s$ depends on the step before the " $\mathrm{D}$ ". If the two previous steps were "LD", then the next step is "R" or "L" according as $s$ is 0 or 1 , while if they were "RD", then the next step is " $\mathrm{L}$ " or " $\mathrm{R}$ " according as $s$ is 0 or 1. For the example of Figure 3, the step sequence is "DRRRDRDLLLDLDRR". 
There are simple algorithms for translating between these two descriptions, and functions that do this are included in the software at [5].

\section{Giant STEPS}

Definition 3.1. Let $\tau$ and $\tau^{\prime}$ be tunnels. We say that $\tau^{\prime}$ is obtained from $\tau$ by a giant step if $\tau$ and $\tau^{\prime}$ are the endpoints of a 1-simplex of $\mathcal{D}(H) / \mathcal{G}$. Equivalently, $\tau$ and $\tau^{\prime}$ can be represented by disjoint disks in $H$.

In 6, Goda, Scharlemann, and Thompson gave a geometric definition of giant steps, which they called tunnel moves, as follows. Let $\tau$ be a nonseparating disk in $H$, and let $K$ be a simple closed curve in $\partial H$ that intersects $\tau$ transversely in one point. Let $N$ be a regular neighborhood in $H$ of $K \cup \tau$. Then the frontier of $N$ separates $H$ into two solid tori, one a regular neighborhood of $K$, so $K$ is a tunnel number 1 knot.

In the previous construction, the meridian disk $\tau^{\prime}$ of the solid torus that does not contain $K \cup \tau$ is the unique nonseparating disk $\tau^{\prime}$ in $H$ that is disjoint from $K \cup \tau$, and $\tau^{\prime}$ is a tunnel of $K$. That is, the construction produces a specific tunnel of the resulting knot $K$. A giant step as we have defined it simply amounts to choosing the $\tau^{\prime}$ first; $K$ is then determined up to isotopy in $H$ and in $S^{3}$, although not up to isotopy in $\partial H$.

Since the complex $\mathcal{D}(H) / \mathcal{G}$ is connected, we have the following, which is part of Proposition 1.11 of [6].

Proposition 3.2. Let $\tau$ be a tunnel of a tunnel number 1 knot. Then there is a sequence of giant steps that starts with the tunnel of the trivial knot and ends with $\tau$.

The depth of a tunnel $\tau$ is defined to be the distance in the 1-skeleton of $\mathcal{D}(H) / \mathcal{G}$ from $\pi_{0}$ to $\tau$. That is, the depth is exactly the length of a minimal sequence of giant steps from $\pi_{0}$ to $\tau$.

\section{Minimal sequences of giant steps}

In this section we give the algorithm to calculate the number of minimal length sequences of giant steps that start from $\pi_{0}$, the tunnel of the trivial knot, and end with a given tunnel $\tau$. This is an elementary combinatorial problem, and Lemma 4.2 will show that it is essentially the problem of computing the number of distinct geodesics between two points in the Farey graph. We will use the algorithm to see that for a sparse infinite set of tunnels, the minimal giant step sequence construction is unique, but generically a tunnel will have many such constructions. In Proposition 4.4, we will characterize the tunnels having a unique minimal giant step sequence.

By a path (between two vertices) in $\mathcal{D}(H) / \mathcal{G}$, we mean a simplicial path in the 1-skeleton of $\mathcal{D}(H) / \mathcal{G}$ passing through a sequence of vertices that are images of vertices of $\mathcal{D}(H)$ (i.e., vertices that represent tunnels). We describe such a path simply by listing the vertices through which it passes. From Section 3 , we know that the minimal sequences of giant steps from $\pi_{0}$ to a given tunnel $\tau$ correspond exactly to the minimal-length paths in $\mathcal{D}(H) / \mathcal{G}$ from $\pi_{0}$ to $\tau$. We will be interested only in minimal-length paths. 

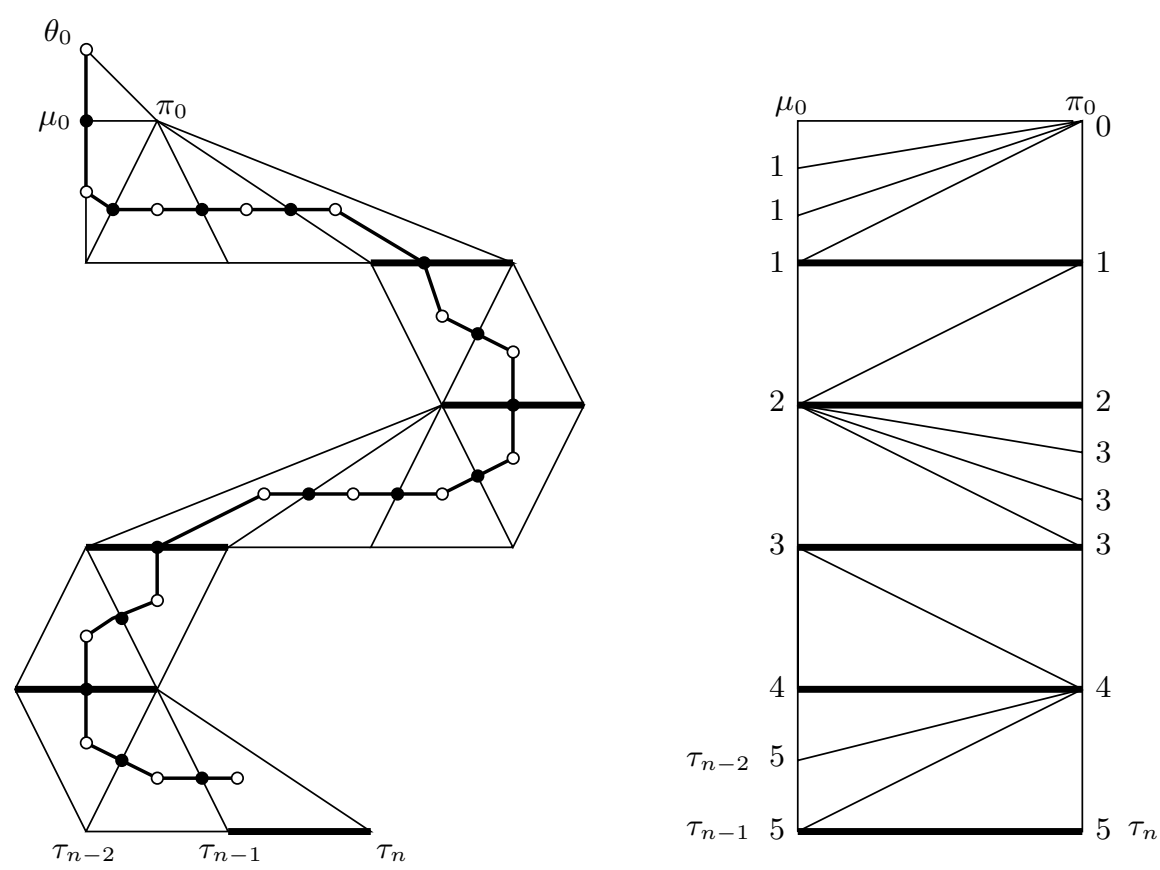

FiguRE 4. The principal path and corridor $C\left(\tau_{n}\right)$ of a tunnel $\tau_{n}$. The $\nabla$-edges are emphasized. In the picture of $C\left(\tau_{n}\right)$ on the right, the depths of the tunnels are labeled.

Definition 4.1. Let $\tau$ be a nontrivial tunnel. Define the corridor of $\tau, C(\tau)$, as follows. Write the vertices of the principal path of $\tau$ as $\theta_{0}, \mu_{0}, \mu_{0} \cup \tau_{0}, \mu_{1}$, $\mu_{1} \cup \tau_{1}, \ldots, \mu_{n} \cup \tau_{n}$, where $\tau=\tau_{n}$. Then $C(\tau)$ is the union of the 2-simplices whose barycenters are the $\mu_{i} \cup \tau_{i}$ for $0 \leq i \leq n$ (where $\mu_{0} \cup \tau_{0}$ is regarded as the barycenter of the 2 -simplex spanned by $\pi_{0}, \mu_{0}$, and $\left.\tau_{0}\right)$.

When $\tau$ is a simple tunnel, $C(\tau)$ is the triangle $\left\langle\pi_{0}, \mu_{0}, \tau_{0}\right\rangle$. Otherwise, it can be viewed as a rectangular or trapezoidal strip with top and bottom edges $\left\langle\mu_{0}, \pi_{0}\right\rangle$ and $\left\langle\tau_{n-1}, \tau_{n}\right\rangle$, as in the drawing on the right in Figure 4 .

Lemma 4.2. Let $\tau$ be a tunnel, and let $\sigma_{0}, \sigma_{1}, \ldots, \sigma_{n}$ be a path in $\mathcal{D}(H) / \mathcal{G}$ of minimal length among the paths connecting the vertices $\sigma_{0}$ to $\sigma_{n}$. If $\sigma_{0}$ and $\sigma_{n}$ lie in $C(\tau)$, then each $\sigma_{i}$ lies in $C(\tau)$.

Proof. If the lemma is false, then there exist $i$ and $j$ with $0 \leq i<i+1<j \leq n$ for which $\sigma_{i}$ and $\sigma_{j}$ lie in $C(\tau)$, but $\sigma_{k}$ does not lie in $C(\tau)$ for any $k$ with $i<k<j$.

The barycenter $\left\{\sigma_{i}, \sigma_{i+1}\right\}$ of the edge $\left\langle\sigma_{i}, \sigma_{i+1}\right\rangle$ lies in the link $L\left(\sigma_{i}\right)$ of $\sigma_{i}$ in $\mathcal{D}^{\prime}(H) / \mathcal{G}$. This link is a tree, so there exists a vertex $\sigma_{i}^{\prime}$ in $C(\tau)$ such that $\left\{\sigma_{i}, \sigma_{i}^{\prime}\right\}$ is connected to $\left\{\sigma_{i}, \sigma_{i+1}\right\}$ by a path in $L\left(\sigma_{i}\right)$ meeting $C(\tau)$ only in $\left\{\sigma_{i}, \sigma_{i}^{\prime}\right\}$. The 1-simplex in $\mathcal{D}(H) / \mathcal{G}$ spanned by $\sigma_{i}$ and $\sigma_{i}^{\prime}$ separates $\mathcal{D}(H) / \mathcal{G}$, with $C(\tau)$ and $\sigma_{i+1}$ lying in different components. Therefore $\sigma_{j}$ must equal either $\sigma_{i}$ or $\sigma_{i}^{\prime}$. In either case we obtain a shorter path from $\sigma_{0}$ to $\sigma_{n}$.

In the special case that $\tau$ is of depth $1, \tau$ lies in the link in $\mathcal{D}(H) / \mathcal{G}$ of $\pi_{0}$, and there is a unique path of length 1 from $\pi_{0}$ to $\tau$. From now on, we assume that $\tau$ has depth at least 2 . 
Now, regard $C(\tau)$ as in the diagram on the right in Figure 4 , with the edge $\left\langle\mu_{0}, \pi_{0}\right\rangle$ on top, and with $\tau$ as one of the endpoints of the bottom edge. In the triangulation of $C(\tau)$, a $\nabla$-edge of depth $i$ is an edge whose endpoints have depth $i$ and lie on different sides of $C(\tau)$, and for which all vertices lying below its endpoints on either side have depth greater than $i$. In Figure 4, the $\nabla$-edges are highlighted.

Since the endpoints of any edge of $C(\tau)$ can have depths that differ by at most 1 , there exists a unique $\nabla$-edge $\nabla(i)$ in $C(\tau)$ of depth $i$ for each $i$ with $1 \leq i<$ $\operatorname{depth}(\tau)$. There is also a $\nabla$-edge for $i=\operatorname{depth}(\tau)$ unless $\tau$ and the endpoints of $\nabla(\operatorname{depth}(\tau)-1)$ span a 2 -simplex.

The name $\nabla$-edge arises from the fact that (except for $\nabla(\operatorname{depth}(\tau)))$ the $\nabla$-edges are the tops of 2-simplices of the corridor that appear as $\nabla$ 's (as opposed to $\Delta$ 's) when the corridor is drawn with depth corresponding to the vertical coordinate, as in the diagram on the left in Figure 4. Every nonprimitive 2-simplex of $\mathcal{D}(H) / \mathcal{G}$ has two vertices of the same depth and a third of depth either larger by 1 or smaller by 1 than that common depth; for a " $\nabla$ " 2 -simplex, that depth is larger by 1 , while it is smaller by 1 for a " $\Delta$ " 2 -simplex.

Denote the left and right endpoints of $\nabla(i)$ by $\partial_{L} \nabla(i)$ and $\partial_{R} \nabla(i)$ respectively.

Lemma 4.3. Let $\nabla(i-1)$ and $\nabla(i)$ be successive $\nabla$-edges. Then at least one of the pairs $\left\{\partial_{L} \nabla(i-1), \partial_{L} \nabla(i)\right\}$ and $\left\{\partial_{R} \nabla(i-1), \partial_{R} \nabla(i)\right\}$ are the endpoints of an edge that lies in a side of $C(\tau)$.

Proof. For each endpoint of $\nabla(i)$, select a path of length $i$ from the endpoint to $\pi_{0}$. By Lemma 4.2 these paths lie in $C(\tau)$. In particular, each of their first edges connects an endpoint of $\nabla(i)$ to an endpoint of $\nabla(i-1)$. At most one of these first edges can be diagonal, so at least one lies in a side.

Lemma 4.3 shows that the triangulation of the portion of $C$ between $\nabla(i-1)$ and $\nabla(i)$ must have one of the four configurations $L_{1}, R_{1}, L_{2}$, or $R_{2}$ shown in Figure 5 , The portion of $C(\tau)$ above $\nabla(1)$ must be as in the leftmost diagram in Figure 5 , perhaps with only one 2 -simplex above the diagonal.

Now we are ready to calculate the number of minimal paths from $\pi_{0}$ to $\tau$. Denote by $\lambda_{i}$ the number of paths in $C(\tau)$ of length $i$ from $\pi_{0}$ to $\partial_{L} \nabla(i)$, and by $\rho_{i}$ the number of paths to $\partial_{R} \nabla(i)$. Clearly $\left(\begin{array}{l}\lambda_{1} \\ \rho_{1}\end{array}\right)=\left(\begin{array}{l}1 \\ 1\end{array}\right)$.

Let $k$ be the maximum $i$ for which $\nabla(i)$ is defined. For each $2 \leq i \leq k$, let $C_{i}$ be $L_{1}, R_{1}, L_{2}$, or $R_{2}$ according to which of the four configurations in Figure 5 describes the triangulation of $C(\tau)$ between $\nabla(i-1)$ and $\nabla(i)$. For $2 \leq i \leq k$, let $M_{i}$ be the matrix given in the following table, according to the value of $C_{i}$ :

\begin{tabular}{|c|c|c|c|c|}
\hline$C_{i}$ & $L_{1}$ & $R_{1}$ & $L_{2}$ & $R_{2}$ \\
\hline \multirow{2}{*}{$M_{i}$} & $\left(\begin{array}{ll}1 & 0 \\
1 & 1\end{array}\right)$ & $\left(\begin{array}{ll}1 & 1 \\
0 & 1\end{array}\right)$ & $\left(\begin{array}{ll}1 & 0 \\
1 & 0\end{array}\right)$ & $\left(\begin{array}{ll}0 & 1 \\
0 & 1\end{array}\right)$ \\
\hline
\end{tabular}

Observe that

Therefore we have

$$
M_{i}\left(\begin{array}{c}
\lambda_{i-1} \\
\rho_{i-1}
\end{array}\right)=\left(\begin{array}{c}
\lambda_{i} \\
\rho_{i}
\end{array}\right)
$$

$$
\left(\begin{array}{c}
\lambda_{k} \\
\rho_{k}
\end{array}\right)=M_{k} M_{k-1} \cdots M_{2}\left(\begin{array}{l}
1 \\
1
\end{array}\right)
$$



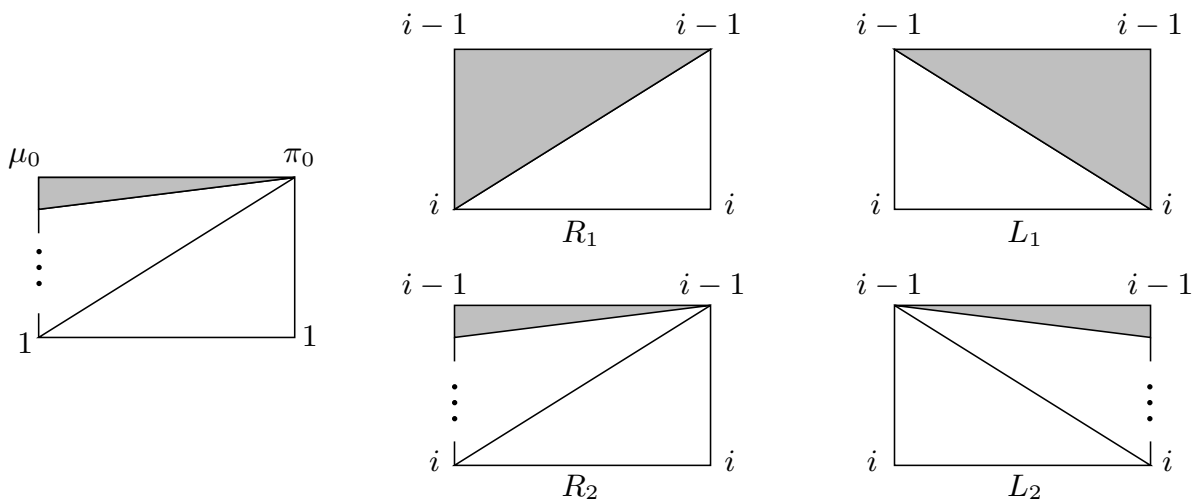

FiguRE 5. The configuration above $\nabla(1)$, and the four possible configurations between two $\nabla$-edges. In $R_{1}$ and $L_{1}$ there is only one 2 -simplex above the diagonal edge, while in $R_{2}$ and $L_{2}$ there are two or more. In the configuration above $\nabla(1)$, there may be only one 2 -simplex above the diagonal. The shaded 2 -simplices are $\nabla 2$-simplices. The letter $L$ (respectively, $R$ ) signifies that the portion below the $\nabla$-simplex contains "L" steps (respectively, "R" steps) of the principal path.

If $\tau$ and the endpoints of $\nabla(k)$ span a 2-simplex, as in the case of the tunnel $\tau_{n-2}$ in Figure 4, then the number of minimal-length paths from $\pi_{0}$ to $\tau$ is $\lambda_{k}+\rho_{k}$. Otherwise, $\tau$ is the left or right endpoint of $\nabla(k)$, and the number of minimal-length paths equals $\lambda_{k}$ or $\rho_{k}$ respectively.

The algorithm just described is not difficult to implement computationally [5]. For the example in Figure 4 , the output of the program is:

Depth> gst ( '0011100011100', verbose = True )

The block configurations are R1, L2, L1, R2, and

M_5 * .. * M_2 $=[[2,2],[2,2]]$.

This tunnel has 4 minimal giant step constructions.

while if we add one downward step at the end:

Depth> gst ( '00111000111001', verbose = True )

The block configurations are R1, L2, L1, R2, and

M_5 * ... * M_2 $=[[2,2],[2,2]]$.

The final block is a nabla.

This tunnel has 8 minimal giant step constructions.

Some examples are the tunnels whose parameter sequences are the following:

(1) $s_{2} s_{3} \cdots s_{n}=100100 \cdots 100$, or DRDLLDRRDLL $\cdots D R R$ (or $\cdots D L L$ ) as a step sequence. The configuration sequence alternates as $L_{2}, R_{2}, L_{2}$, $R_{2} \ldots$, and there is a unique minimal giant step sequence.

(2) $s_{2} s_{3} \cdots s_{2 n+1}=1010 \cdots 10$, or $D R D L D R D L D \cdots D R$ (or $\left.\cdots D L\right)$. The configuration sequence alternates as $L_{1}, R_{1}, L_{1}, R_{1} \ldots$, and the number of minimal giant step sequences is the term $F_{n}$ of the Fibonacci sequence $\left(F_{0}, F_{1}, F_{2}, \ldots\right)=(1,1,2,3,5, \ldots)$.

(3) $s_{2} s_{3} \cdots s_{2 n+1}=111 \cdots 1$, an even number of 1's, or DRDRDR $\cdots D R$. The configuration sequence is $R_{1}, R_{1}, \ldots, R_{1}, \tau$ is the right-hand endpoint of $\nabla(n)$, and there is a unique minimal giant step sequence. 
(4) $s_{2} s_{3} \cdots s_{2 n}=111 \cdots 1$, an odd number of 1's, or $D R D R D R \cdots D$. The configuration sequence is again $R_{1}, R_{1}, \ldots, R_{1}$, but $\tau$ lies in a $\nabla 2$-simplex below $\nabla(n)$, and there are $n+1$ minimal giant step sequences.

Examples of the last two types are obtained from each other by a single additional cabling construction, even though the numbers of minimal giant step constructions differ by arbitrarily large amounts.

We can characterize the tunnels that have a unique minimal giant step sequence. In part (c) of the next proposition, $C_{i}$ denotes the configuration between $\nabla(i-1)$ and $\nabla(i)$ in the corridor $C(\tau)$ as shown in Figure 4 .

Proposition 4.4. Let $\tau$ be a tunnel of a nontrivial tunnel number 1 knot, with $\tau$ not simple. Then $\tau$ has a unique minimal giant step sequence if and only if it satisfies one of the following three equivalent conditions:

(a) Its step sequence does not end in " $D$ " and does not contain as a block either "DLDR" or a noninitial "DRDL".

(b) Its binary sequence, broken into blocks of 0's and blocks of 1's, does not end in a block of 1's of odd length and does not contain a block of either of the forms $[1 \cdots 1] 1110$ or $[1 \cdots 1] 1010$, where $[1 \cdots 1]$ denotes a block of even (possibly zero) length.

(c) It is an endpoint of the edge $\nabla(k)$, where $k$ is the depth of $\tau$, and in the descending sequence of configurations $C_{1} C_{2} \cdots C_{k}$ of the corridor, no $C_{i} C_{i-1}$ is in $\left\{L_{1} R_{1}, L_{2} R_{1}, R_{1} L_{1}, R_{2} L_{1}\right\}$ for $3 \leq i \leq k$.

Proof. We begin with part (c). When $k=1$, there is a unique edge connecting $\tau$ to $\pi_{0}$, and the condition in (c) is true. Suppose that $k=2$. If $\tau$ is not an endpoint of $\nabla(2)$, then $\tau$ and the endpoints of $\nabla(1)$ bound a $\nabla 2$-simplex, there are two minimal-length paths, and the condition in (c) is false. If $\tau$ is an endpoint of $\nabla(2)$, then there is a unique edge from $\tau$ to a depth- 1 tunnel, and consequently a unique path of length 2 from $\tau$ to $\pi_{0}$. Condition (c) is true, its latter part vacuously, so again it gives the correct characterization.

Suppose now that $k \geq 3$. As before, $\lambda_{i}$ will denote the number of paths in $C(\tau)$ of length $i$ from $\pi_{0}$ to the left endpoint $\partial_{L} \nabla(i)$, and $\rho_{i}$ will denote the number of paths to $\partial_{R} \nabla(i)$. We may assume that $\tau$ is an endpoint of $\nabla(k)$, since otherwise $\tau$ is connected by edges to both endpoints of $\nabla(k-1)$, in which case the number of minimal sequences is $\lambda_{k-1}+\rho_{k-1}>1$ and (c) is false. In the sequence of tunnels $\tau_{j}$ in Definition 4.1, let $\sigma_{i}$ be the last one at depth $i$, in particular, $\tau=\sigma_{k}$. Let $N_{i}$ be the number of distinct minimal length paths from $\sigma_{i}$ to $\pi_{0}$, in particular, $N_{1}=N_{2}=1$.

We will induct on $k$, showing in the process that $N_{i}=\min \left\{\lambda_{i}, \rho_{i}\right\}$, that $N_{i-1} \leq$ $N_{i}$, and that $N_{i-1}<N_{i}$ only when $C_{i} C_{i-1}$ is one of the four combinations listed in (c). Examining the configuration $C_{k-1}$, we have four cases:

(L1) $C_{k-1}=L_{1}, \sigma_{k-1}=\partial_{L} \nabla(k-1)$, so $N_{k-1}=\lambda_{k-1}$, and $\lambda_{k-1}<\rho_{k-1}$,

(R1) $C_{k-1}=R_{1}, \sigma_{k-1}=\partial_{R} \nabla(k-1)$, so $N_{k-1}=\rho_{k-1}$, and $\rho_{k-1}<\lambda_{k-1}$,

(L2) $C_{k-1}=L_{2}, \sigma_{k-1}=\partial_{L} \nabla(k-1)$, so $N_{k-1}=\lambda_{k-1}$, and $\lambda_{k-1}=\rho_{k-1}$, or

(R2) $C_{k-1}=R_{2}, \sigma_{k-1}=\partial_{R} \nabla(k-1)$, so $N_{k-1}=\rho_{k-1}$, and $\lambda_{k-1}=\rho_{k-1}$.

For the inductive step, suppose first that $C_{k}=L_{1}$. Examining the configuration $L_{1}$, we see that $\tau=\partial_{L} \nabla(k)$ and $N_{k}=\lambda_{k}=\lambda_{k-1}$. Also, $\lambda_{k}<\lambda_{k-1}+\rho_{k-1}=\rho_{k}$, so $N_{k}=\min \left\{\lambda_{k}, \rho_{k}\right\}$. In cases (L1) and (L2), $N_{k-1}=\lambda_{k-1}=N_{k}$, and in case (R2), $N_{k-1}=\rho_{k-1}=\lambda_{k-1}=N_{k}$. In case (R1), which produces $C_{k} C_{k-1}=L_{1} R_{1}$ and 
makes (c) false, we have $N_{k-1}=\rho_{k-1}<\lambda_{k-1}$, so $N_{k-1}<N_{k}$ and $\tau$ does not have a unique minimal giant step sequence. This completes the induction when $C_{k}=L_{1}$. If $C_{k}=R_{1}$, the argument is analogous.

Suppose that $C_{k}=L_{2}$. Then again, $\tau=\partial_{L} \nabla(k-1)$ and $N_{k}=\lambda_{k}=\lambda_{k-1}$, but this time $\rho_{k}=\lambda_{k-1}=\lambda_{k}$. Again, $N_{k}=\min \left\{\lambda_{k}, \rho_{k}\right\}$. Case (R1) gives $N_{k-1}<N_{k}$, and the other cases give $N_{k-1}=N_{k}$, and again $C_{k}=R_{2}$ is analogous. This completes the induction and the proof of part (c).

Part (a) now follows, since the sequences "DLDR" and a noninitial "DRDL" are exactly those which produce the four pairs listed in (c) for $C_{i} C_{i-1}$. For part (b), observe that in a block of 1's, the first, third, and so on correspond to downward steps, and the others to horizontal steps. The four pairs in part (c) correspond exactly to blocks of the form 1(0 or 1)10 with the initial 1 a downward step, and this gives (b).

Proposition 4.4 shows that tunnels having more than one minimal giant step sequence are generic in any reasonable sense.

\section{REFERENCES}

1. E. Akbas, A presentation of the automorphisms of the 3-sphere that preserve a genus two Heegaard splitting, Pacific J. Math. 236 (2008), 201-222. MR2407105 (2009d:57029)

2. S. Cho, Homeomorphisms of the 3-sphere that preserve a Heegaard splitting of genus two, Proc. Amer. Math. Soc. 136 (2008), 1113-1123. MR2361888(2009c:57029)

3. S. Cho and D. McCullough, The tree of knot tunnels, Geom. Topol. 13 (2009), 769-815. MR2469530

4. S. Cho and D. McCullough, Tunnel leveling, depth, and bridge numbers, arXiv:0812.1396.

5. S. Cho and D. McCullough, software available at www.math.ou.edu/ dmccullough/research/ software.html .

6. H. Goda, M. Scharlemann, and A. Thompson, Levelling an unknotting tunnel, Geom. Topol. 4 (2000), 243-275. MR1778174 (2002h:57011)

7. M. Scharlemann, Automorphisms of the 3-sphere that preserve a genus two Heegaard splitting, Bol. Soc. Mat. Mexicana (3) 10 (2004), 503-514. MR2199366 (2007c:57020)

8. M. Scharlemann and A. Thompson, Unknotting tunnels and Seifert surfaces, Proc. London Math. Soc. (3) 87 (2003), 523-544. MR1990938(2004e:57015)

Department of Mathematics, University of California at Riverside, Riverside, CaliFORNIA 92521

E-mail address: scho@math.ucr.edu

Department of Mathematics, University of Oklahoma, Norman, Oklahoma 73019

E-mail address: dmccullough@math.ou.edu 